\title{
Calculation of Drug Dosage In Chronic Kidney Disease
}

\author{
Tenta Hartian Hendyatama ${ }^{1}$, Nunuk Mardiana ${ }^{2}$ \\ ${ }^{1}$ Department of Internal Medicine, Faculty of Medicine, Universitas Airlangga - Dr. Soetomo General Hospital Surabaya, \\ Indonesia. \\ ${ }^{2}$ Division of Nephrology and Hypertension, Department of Internal Medicine, Faculty of Medicine, Universitas Airlangga - \\ Dr. Soetomo General Hospital Surabaya, Indonesia.
}

\begin{abstract}
A B S T R A C T
Kidneys are the main organ in fluid and electrolyte homeostasis. It also have an important role in eliminating various types of drugs. Drug elimination in the kidney is affected by plasma drug concentrations, plasma protein binding, and kidney function. Glomerular filtration rate (GFR) represents the kidney function. Thus by knowing it, drug dosage can be determined. Chronic kidney disease alter the effect of drug, some decrease drug effect but more often increase drug toxicity. Chronic kidney disease affect the pharmacodynamic and pharmacokinetic of drug. Therefore, providing an optimal treatment for CKD patient, knowledge of the pharmacokinetic and pharmacodynamic changing in CKD is needed. Exploring the patient's history and carrying out complete physical examination is important before giving the drug to patients with impaired kidney function. In addition, identifying drugs that have the potential to cause nephrotoxicity and drug interactions is also important. The body response to drugs in patient with CKD is very varied, complex, and individual. Dosage must be based on several factors, not only glomerular filtration rates but also other comorbid diseases, interactions with other drugs and clinical condition of the patient.
\end{abstract}

Keywords: : Drug, dosage, Chronic Kidney Disease, Pharmacokinetic, Pharmacodynamic

Correspondence: Nunuk Mardiana

E-mail: nunuk.mardiana@yahoo.com

Article history: • Received 26 December $2019 \bullet$ Received in revised form 09 January 2020• Accepted 16 January 2020

\section{INTRODUCTION}

Kidneys are the main organ in fluid and electrolyte homeostasis. It also have an important role in eliminating various types of drugs. Drug elimination in the kidney is affected by plasma drug concentrations, plasma protein binding, and kidney function. Glomerular filtration rate (GFR) represents the kidney function. Thus by knowing it, drug dosage can be determined (Alahdal and Elberry, 2012, Matzke et al., 2011, Munar and Singh, 2007).

According to Basic Health Research (Riskesdas), the prevalence of chronic kidney disease (CKD) in Indonesia is about $0.2 \%$. The prevalence of age $>75$ years is $0.6 \%$ higher than people under 75 years. In the last ten years, prevalence of CKD has increased throughout the world. In 2013, the growth of CKD increased by $50 \%$ from the previous year (Hill et al., 2016).

Chronic kidney disease alter drug effect. Some of them decrease drug effect but more often increase drug toxicity. Therefore, providing an optimal treatment for CKD patient, knowledge of the pharmacokinetic and pharmacodynamic changing in CKD is needed.(Chan SK, 2009, Doogue and Polasek, 2011) In this paper, the regulation of drug dosage in chronic kidney disease will be explained including : glomerular filtration rate, pharmacokinetics and pharmacodynamics changing in CKD, calculation of drug dosage in CKD and drug monitoring in $\mathrm{CKD}$.

\section{Glomerular Filtration Rate}

The kidney has about 1 million nephrons. Each nephron produces a filtrate that is affected by plasma flow, gradient pressure, surface area, and glomerular filtration barrier permeability. The assessment of kidney function that is often used is glomerular filtration rate because glomerulus act as a filter (Miles, 2013).

Glomerular filtration rate is the amount of substances or drugs in plasma which are completely filtered into the urine per unit time. Ideal substances for calculating glomerular filtration rate are freely filtered through the glomerulus and neither absorbed, metabolized nor secreted along nephron (Hudson and Nyman, 2011, Jones, 2011). Substance which is used for calculating glomerular filtration rate are inulin, creatinine and cystatin C. Creatinine is the most widely used for estimating kidney function because creatinine is endogenously produced, cheap and easy to measure. This calculation needs 24-hour urine specimens and plasma creatinine which is measured on the same day.

\section{Estimated Glomerular Filtration Rate}

Creatinine clearance calculation by collecting 24 hours urine certainly takes a long time. So, national and international organizations recommend calculating estimated GFR 
(eGFR) by using creatinine based estimated formula as the first step in estimating kidney function.(Miles, 2013) Some estimation formulas for eGFR are Cockroft-Gault, Modification of Diet in the Renal Disease Study Equation (MDRD), Chronic Kidney Disease Epidemiology Collaboration (CKD-EPI) Formula. Cockroft-Gault Formula is the most widely used for estimating GFR. Mathematically:

\section{(( 40-Age (Year))x Weight (Kg)x 0.85 (in women)) / (72 x Serum Creatinine)}

\section{The Effect Of Chronic Kidney Disease on Pharmakokinetics}

Chronic kidney disease is a clinical syndrome of the persistent or irreversible kidney function declining which is characterized by glomerular filtration rate declining. It runs progressively and chronically become an End Stage Renal Disease (ESRD) (Lam et al., 1997, Vassalotti et al., 2016).

Chronic kidney disease (CKD) can affect the pharmacokinetics of a drug. Pharmacokinetics is the way of the body acts on the drug once it is administered including : absorption, distribution, metabolism and elimination. CKD affects : (1) absorption and bioavailability; (2) distribution volume and protein binding; (3) metabolism; (4) elimination (Chan SK, 2009).

\section{Absorption and Bioavailability}

Bioavailability is the percentage of the active substance of drug that reaches the systemic circulation after drug administration. Before drugs have systemic effects, they must be absorbed into the body and metabolized into their active form. Bioavailability can be affected in patients with renal failure. Vomiting and impaired peristalsis due to uremic enteropathy may reduce the drug absorption rate. Drugs commonly used in renal failure, including phosphate binders and proton pump inhibitors, reduce acidic drug absorption; while phosphate binders also form a complex with certain antibiotics and iron tablets. Renal failure frequently causes gastrointestinal tract edema, which further affects drug absorption (Chan SK, 2009).

\section{Distribution Volume and Protein Binding}

Drug distribution is process of transferring drug from the systemic to interstisial and tissue cells. Distribution volume is the volume needed to load the amount of drug in the plasma. Distribution volume describes the broad distribution of drugs in the body. The higher the distribution volume, the higher the concentration of the drug in extravascular tissue.

A number of factors can affect drug's distribution volume. These include body size, age, gender, renal function, protein binding, and presence of other drugs. A decrease in protein binding causes an increase in distribution volume. Renal impairment induces a decrease in the ability of plasma proteins to bind certain drugs, especially for acidic drugs existing as anions in blood. The possible mechanisms include a reduction in binding protein concentration, competitive displacement from normal binding sites in tissue, or synthesis of a protein with reduced binding sites. For drugs that are normally highly proteinbound to plasma proteins, small changes in the extent of binding due to renal impairment produce a large change in the amount of unbound drugs, and hence susceptibility to toxicity for a drug with a narrow therapeutic index.

Hypoalbuminemia, commonly seen in renal failure, may increase the free fraction of drugs appearing in the plasma so drug efficacy and toxicity increase. Conversely, an increased binding can lead to reduced pharmacologic effects on the therapeutic concentration (Miles, 2013).

\section{Drug Metabolism}

Most drugs undergo biotransformation to metabolites, which are then excreted. The usual pathways of drug metabolism include oxidation, reduction, and hydrolysis. The renal component of drug clearance will be reduced in renal failure patients, while hepatic metabolism may be increased as a compensatory mechanism. Hepatic metabolism of some drugs, however, will be slower in renal failure patients (e.g. propranolol oxidation, hydrocortisone reduction, cephalosporin hydrolysis).

In CKD, excretion of drug metabolites will decrease so that it can lead to accumulation of drug metabolites. If the drug metabolites are still active it will increase the risk of toxicity. In CKD, uremia also occurs which can cause inactivation of the cytochrome P450 enzyme (CYP) (Chan SK, 2009, Olyaei AJ, 2016).

\section{Drug Elimination}

Drug elimination decrease in patients with CKD. It causes the body to carry out compensation mechanisms by increasing drug metabolism in the liver. Drug elimination in the kidneys depends on glumerular filtration, tubular secretion, reabsorption and renal epithelial cell metabolism. Glomerular filtration certainly changes in patients with CKD. While tubular secretion and reabsorption will be affected by decreased renal blood flow (Chan SK, 2009).

Increasing the clearance of a drug will result in a decrease in the concentration of a drug in the body, while a decrease in the clearance of a drug will result in an increase in the concentration of a drug and certainly will increase the risk of toxicity of a drug. To avoid the toxicity of a drug caused by decreased clearance of a drug, the dose of drugs excreted through the kidneys must be reduced in patients with CKD (Doogue and Polasek, 2011).

\section{EffectOfChronicKidney DiseaseOnPharmacodynamics}

Pharmacodynamics is the biological effect produced by a drug on the body. The study of the effect of CKD on drug pharmacodynamics is still very limited. Some studies conclude that patients with CKD will be more sensitive to some drugs (Quan DJ, 2013).

The uremia condition in CKD will cause changes in the permeability of the blood-brain barrier so that the levels of drugs that work in the central nervous system increase. One example is the administration of morphine in CKD will further increase the effect of suppression on the central nervous system. Another example is the dose of nifedipine should be adjusted because of the increased antihypertensive effect in CKD. Giving spironolactone in CKD will increase the risk of hyperkalemia (Quan DJ, 2013). 


\section{Calculation of Drug Dosage}

Drug dosage's unit is the number of drugs per unit time, for example, $500 \mathrm{mg}$ twice daily. Most of drugs have standard dose recommendations and instructions for taking the dose. However, those guidelines of most drugs are imprecise, it can differ depending on the clearance and response of each individual (Doogue and Polasek, 2011).

Estimated glomerular filtration rate are used to estimate kidney function, and also used to estimate drug clearance through the kidneys. Wholly or partly active ingredient of a drug is cleared from the body through the kidneys. Based on this function of kidney, it is very rational to use eGFR to estimate drug clearance. Similar to serum drug levels, serum creatinine also depends on creatinine production (equivalent to drug dose) and creatinine clearance (equivalent to renal drug clearance) (Doogue and Polasek, 2011).

Drug clearance (CL) and bioavailability (F) (Fraction or parts from drugs that reach the systemic circulation) determine the steady state concentration of the drug in plasma (Cp) in a given dose. CL has a volume / unit time and the F unit is Percent (\%) (Doogue \& Polasek, 2011). It should be noted that CL is not the same as drug elimination.

Drug dosage $=\mathrm{Cp} \times \mathrm{CL} / \mathrm{F}$ with units: $\mathrm{mg} /$ hour $=\mathrm{mg} / \mathrm{L}$ $\mathrm{x} \mathrm{L} /$ hour

From this equation we can see, if CL is halved, the dose of the drug must be halved to reach the same drug concentration. So if a drug is $100 \%$ cleared by kidney and kidney function decreases half-normal, the dose of the drug must be halved. But many of drugs inactivated by metabolism of liver, hence these drugs do not require dosing adjustment for patients with impaired kidney function (Doogue and Polasek, 2011).

Some drugs are partially cleared by the kidneys and some are metabolized in the liver (Example: LMWH). For these drugs, the regulation of drug dosage is less than drug that $100 \%$ cleared by kidney. For example, in patients with half-normal kidney function, the dose of the drug which is partly cleared by kidneys and half metabolized by liver, should be reduced by a quarter (Doogue and Polasek, 2011).

\section{Loading Dose}

In patients with normal kidney function, steady state concentration in the blood will be achieved approximately 3.3 times the half-life of the drug. Steady state concentration is a condition when the rate of entry drugs is equal to the rate of drugs when it is released. Loading dose is used to achieve the desired drug concentration close to the steady state concentration (Olyaei AJ, 2016).

In patient with CKD, the half-life of drug is elongated. So, in general, CKD patient must obtain the same loading dose as patients with normal kidney function to achieve a rapid therapeutic dose. CKD patients who treated with digoxin must be prescribed only half or three quarters of the loading dose, because distribution volume $(\mathrm{Vd})$ from digoxin is diminished in CKD (Olyaei AJ, 2016). The following formula can be used to determine the loading dose.

$$
\begin{aligned}
& \text { LD }=\text { Vd x IBW x Cp } \\
& \text { LD: Loading Dose } \\
& \text { Vd: Distribution volume }(\mathrm{L} / \mathrm{kg}) \\
& \text { IBW: ideal body weight } \\
& \text { Cp: desired plasma concentration }(\mathrm{mg} / \mathrm{L})
\end{aligned}
$$

\section{Maintenance Dose}

To determine the maintenance dose for CKD patients, it is necessary to know the value of the fraction excreted unchanged (fe) of the drug. Fraction excreted unchanged (fe) is the percentage of active drugs that are cleared by the kidneys in healthy people. Drug dosages with fe $\geq 0.5(50 \%$ or more cleared by the kidneys) must be reduced in patients with impaired renal function (Doogue and Polasek, 2011). Following is the formula to calculate maintenance dose in CKD.

Patient Dose $=$ Usual Dose $\mathrm{x}((1-\mathrm{fe})+\mathrm{fe} \mathrm{x}$ estimated patient renal function) / Normal renal function

\section{Antibiotic Dose in Chronic Kidney Disease}

There are 2 pharmacodynamic characteristics of antibiotics which are time-dependent (interval dependent) and concentration-dependent (dose dependent). Timedependent antibiotics will have the optimum bactericidal effect, if the blood drug concentration is maintained above the minimum inhibitory concentration (MIC) or the minimum dose needed to inhibit bacterial growth. The concentration of drugs that is required approximately 2 to 4 times of MIC is achieved by repeating its dose at certain intervals. Increasing dosage of the drug will not enhance the bactericidal effect. Adjustment of time-dependent antibiotic drugs on CKD is by reducing the drug dosage at a fixed interval. Dosage calculation of this type of drug is using the maintenance dose calculation formula. Examples of time-dependent antibiotic is ciprofloxacin (Olyaei AJ, 2016, Levison, 2004).

Concentration-dependent antibiotics will have an optimal bactericidal effect if the concentration in the blood reaches at least 10 times of MIC. The higher drug's concentration in blood, the higher bacterial capacity. This type of antibiotic has post antibiotic effect (PAE), which is the bactericidal ability still present even though blood concentration of drugs is below the MIC. Regulation of concentration-dependent antibiotic drug dosage is to extend interval of drug's administration without reducing the dose. Examples of concentration-dependent antibiotic is levofloxacin (Levison, 2004, Olyaei AJ, 2016). Following is the formula for drug interval adjustment in concentration-dependent antibiotics:

Desired interval $=$ normal eGFR $\mathrm{x}$ normal interval of drug / patient's eGFR 


\section{Dialysis And Drug Clearence}

Most drugs are excreted via dialysis. Dosage adjustment and schedule for drug administration must be considered in patients who will undergo dialysis. Drug should be given after dialysis is finish. Nevertheless, additional dosage should be given if this condition is not possible. Especially in dialysis that generate increasing of total drug clearance $>30 \%$. Molecular weight, drug solubility in water, and binding ability with proteins are the main factors of drugs being dialyzed or not. Afterwards the others that influence are the volume of non-renal distribution and excretion. Moreover, factors of dialysis component also make an impact to drug clearance, such as: dialysis rate, dialysis membrane, temperature, and $\mathrm{pH}$ (Olyaei AJ, 2016).

\section{Drug Level Monitoring}

Patients with CKD have an increased risk of drug toxicity, even though physician has modified drug dosage and interval of drug administration. However, drug levels monitoring is also important, particularly it should be performed on drugs that have narrow therapeutic index such as digoxin and warfarin (Olyaei AJ, 2016).

Monitoring drug levels is expensive and is not always possible everywhere. Subsequently it also does not guarantee reducing the incidence of toxicity. For example, aminoglycosides can be concentrated in tissues, such as the inner ear and renal cortex, which certainly will not be described by high levels of drugs in the blood, so it is necessary to do clinical assessment while prescribing any drug to patient (Olyaei AJ, 2016).

\section{CONCLUSION}

Chronic kidney disease alter drug effect. Some of them decrease drug effect but more often increase drug toxicity. Therefore, providing an optimal treatment for CKD patient, knowledge of the pharmacokinetic and pharmacodynamic changing in CKD is neededExploring the patient's history and carrying out complete physical examination is important before giving the drug to patients with impaired kidney function. In addition, identifying drugs that have the potential to cause nephrotoxicity and drug interactions is also important. The body response to drugs in patient with CKD is very varied, complex, and individual. Dosage must be based on several factors, not only glomerular filtration rates but also other comorbid diseases, interactions with other drugs and clinical condition of the patient.

\section{CONFLICT OF INTEREST}

The author declare there is no conflict of interest of this study.

\section{REFERENCES}

Alahdal, A. M. \& Elberry, A. A. 2012. Evaluation Of Applying Drug Dose Adjustment By Physicians In Patients With Renal Impairment. Saudi Pharmaceutical Journal, 20, 217-220.
Chan Sk, C. L. 2009. Drug Doses In Patients With Renal Impairment. In: Lai, K. N. (Ed.) Practical Manual Of Renal Medicine Nephrology, Dialysis And Transplantation. Singapore: World Scientific Publishing Co. Pte. Ltd.

Doogue, M. P. \& Polasek, T. M. 2011. Drug Dosing In Renal Disease. The Clinical Biochemist Reviews, 32,69 .

Hill, N. R., Fatoba, S. T., Oke, J. L., Hirst, J. A., O'callaghan, C. A., Lasserson, D. S. \& Hobbs, F. R. 2016. Global Prevalence Of Chronic Kidney Disease-A Systematic Review And Meta-Analysis. Plos One, 11, E0158765.

Hudson, J. Q. \& Nyman, H. A. 2011. Use Of Estimated Glomerular Filtration Rate For Drug Dosing In The Chronic Kidney Disease Patient. Current Opinion In Nephrology And Hypertension, 20, 482-491.

Jones, G. R. 2011. Estimating Renal Function For Drug Dosing Decisions. The Clinical Biochemist Reviews, 32, 81.

Lam, Y. F., Banerji, S., Hatfield, C. \& Talbert, R. L. 1997. Principles Of Drug Administration In Renal Insufficiency. Clinical Pharmacokinetics, 32, 30-57.

Levison, M. E. 2004. Pharmacodynamics Of Antimicrobial Drugs. Infectious Disease Clinics, 18, 451-465.

Matzke, G. R., Aronoff, G. R., Atkinson Jr, A. J., Bennett, W. M., Decker, B. S., Eckardt, K.-U., Golper, T., Grabe, D. W., Kasiske, B. \& Keller, F. 2011. Drug Dosing Consideration In Patients With Acute And Chronic Kidney Disease-A Clinical Update From Kidney Disease: Improving Global Outcomes (Kdigo). Kidney Internation$a l, 80,1122-1137$.

Miles, C. 2013. Assesment Of Renal Function. In: Lerma, R. (Ed.) Clinical Decisions In Nephrology, Hipertension And Kidney Transplantation. Usa: Springer.

Munar, M. Y. \& Singh, H. 2007. Drug Dosing Adjustments In Patients With Chronic Kidney Disease. American Family Physician, 75.

Olyaei Aj, F. T., Lerma Ev 2016. Drug Dosing In Chronic Kidney Disease. In: N Turner, N. L., Dj Goldsmith, Cg Winearls, J Himmelfarb, G Remuzzi (Ed.) Oxford Textbook Of Clinical Nephrology, 4th Edition. United Kingdom: Oxford University Press.

Quan Dj, A. F. 2013. Dosing Of Drugs In Renal Failure. In: B Alldredge, R. C., M Ernst, B Guglielmo (Ed.) Applied Therapeutics The Clinical Use Of Drugs. Usa: Lippincott Williams \& Wilkins.

Vassalotti, J. A., Centor, R., Turner, B. J., Greer, R. C., Choi, M., Sequist, T. D. \& Initiative, N. K. F. K. D. O. Q. 2016. Practical Approach To Detection And Management Of Chronic Kidney Disease For The Primary Care Clinician. The American Journal Of Medicine, 129, 153162. E7. 